dementia is poorly defined for the general reader. If the number of pages per field of emphasis reflects relative importance, then the book stresses certain areas very heavily and ignores other major themes of neurobiology related to the dementias.

Any multi-authored compilation runs the risk of being unbalanced. The title of this book implies a cohesive and balanced overview of the neurobiology of dementia. Indeed, several of the chapters are excellent and provide relative placement within the field and an interesting and thoughtful perspective on dementia. The chapter "Calcium Homeostasis and Free Radical Metabolism as Convergence Points in the Pathophysiology of Dementia," is one of the longer contributions and should be read by anyone interested in this field. "Prion Diseases and Dementia" provides an excellent clinical, pathological and genetic overview of these diseases. However, several of the chapters concentrate on authors' pet theories, giving very short shrift to other considerations.

The read is easier if one is already familiar with the field of dementia. A fivepage, well-written summary of the anatomy of $\mathrm{AD}$ may be sufficient for a reader immersed in the field, but a more complete comparative anatomy of $\mathrm{AD}$ and other dementias may be more helpful to the general audience. There are no fewer than five chapters that deal with the role of the amyloid $\beta$-protein $(A \beta)$ precursor (APP) or the putative effects of $A \beta$. Each of these chapters is based on the fundamental hypothesis that the deposition of the A $\beta$ fragment of APP causes AD. Although this reflects the interests of the editors, it is not balanced by inclusion of proponents of several alternative viewpoints on pathogenesis. The editors state that "the contribution of genetic strategies has been fundamental to discovery," and they are correct. However, most of AD is not due to autosomal dominant mutations (defined or remaining unknown) and, for example, a growing field of neurobiology related directly to the brain metabolism of apolipoprotein $\mathrm{E}$ is summarized in a single chapter with one point of view.

Gene discovery in the defined forms of $\mathrm{AD}$ also provides a means of predicting which groups of patients are most likely to become afflicted with AD. A growing and important literature germane to discussions of molecular mechanisms is emerging from the combined application of genetics and epidemiology to temporal questions of disease onset and pathogenesis. It is certainly difficult to tell what occurs first when only post-mortem material is considered. However, it is now possible to predict which groups of individuals are at risk and to apply a range of metabolic and anatomical analyses to study their temporal relationship with onset of dementia. For example, positron emission tomography studies of groups of unaffected individuals who are at an increased genetic risk for $\mathrm{AD}$ have documented decreased oxygen metabolism in areas of the brain characteristic of $\mathrm{AD}$. These metabolic changes occur more than two decades before the median age of onset of disease, well before significant $A \beta$ deposition has occurred. Unfortunately, the role of genetics combined with functional imaging, earliest neuropathology, epidemiology and other exciting emerging fields is barely mentioned in this text.

The book contains several superb chapters by authors with defined viewpoints and these are interesting and provocative reading for scientists in the field of dementia. However, as a general reference to be read cover to cover by interested neuroscientists, the volume is unbalanced. No book of this length could hope to be complete, but a balanced distribution of content within context would make the book more accessible to the general neurobiologist.

\section{Concepts in Vaccine Development}

\section{edited by Stefan H.E. Kaufmann Walter de Gruyter \& Co. ISBN: 3-11-014815-3 \$119.00}

REVIEWEd BY LOUIS D. FALO, JR. Assistant Professor and Vice Chairman Department of Dermatology

University of Pittsburgh School of Medicine

University of Pittsburgh Cancer Institute

Pittsburgh, Pennsylvania 15213, USA

Recent advances in several disciplines have resulted in significant progress in vaccine design. "Vaccinology" has evolved from a primarily empirical art to a hypothesis-driven science drawing on advances in molecular genetics, cell biology, protein chemistry and immunology. Vaccine design is now the focus of multidisciplinary teams of investigators in academia and of "core technology platforms" in the private sector. It is perhaps this influx of new investigators and the convergence of established investigators with diverse scientific backgrounds that has created a need for a book that can serve as a single-source, comprehensive overview of vaccinology. Concepts in Vaccine Development, successfully addresses this need.

This book aims to provide the reader with a state-of-the-art overview of "all aspects of vaccinology from the bench to the field." As a collection of reviews, its breadth is certainly impressive, beginning with a bicentennial tribute to the first vaccination by Edward Jenner and ending with a lucid review of the application of vaccination therapy to autoimmune disease. The 53 contributors (to 22 chapters) provide a systematic review of critical issues in a nearly manageable 570 pages. As is the case with most books of this type, individual chapters vary in quality. The inclusion of so many topics means that several chapters are somewhat limited in scope. Similarly, it is rare for such books to offer the most up-to-date information, particularly in areas where progress has been so rapid. However, the real strength of this text is not in the chapters dealing with subjects with which the reader may be familiar, but in the chapters on less familiar topics. Most would serve as excellent starting points for ventures into new areas of investigation. Most important, by presenting quality reviews on diverse concepts, this book provides the reader with a genuine sense of perspective.

The book is divided into four sections. The first appropriately establishes the need for new vaccines, addressing the issue from evolutionary, economic and public health perspectives. A review addressing the economics of vaccination presents economic concepts and analysis in a manner amenable to non-economists and includes a particularly compelling discussion of the use of "cost-effectiveness" analysis to identify priorities in vaccine research.

The other three sections are oriented more toward basic research, and the reader can readily sense the renewed optimism that has permeated this field. The section "General Principles of Immunology" introduces concepts in the context of strategies for vaccine design against intracellular bacteria, viruses and Leishmania parasites. The chapter on viral vaccine strategies ends with a general consideration of cytotoxic T-lymphocyte priming and immunodominance and nicely complements the fol- 
lowing chapter discussing broadly reactive T-cell epitopes and their implications for vaccine design.

The book then moves to a discussion of general principles of vaccinology, beginning with a well-written overview of modern adjuvants. This section is organized primarily by methods of antigen delivery and includes the development of peptide-based vaccines and the use of biodegradable polymers for protein antigen delivery. Recent developments in genetic approaches for vaccine delivery are introduced, including the use of recombinant bacteria, recombinant viruses and naked DNA for antigen delivery. While individual chapters are well done, the reader is left hanging without a unifying discussion of potential mechanisms of immunization or rationale for the pursuit of one recombinant strategy versus another. Also noticeably absent from this section is any discussion of the potential use of dendritic cells as vehicles for antigen delivery, either through adoptive transfer or by in vivo targeting.

The book concludes with a series of reviews on vaccination strategies against

\section{Between Bench and Bedside:}

\section{SCIENCE, HEALING AND INTERLEUKIN-2 IN A CANCER WARD \\ by Ilana Lowy \\ Harvard University Press \\ 1997 ISBN: 0-674-06809-2 \$39.95}

\section{REVIEWED By RALPH A. REISFELD}

Head, Division of Tumor Cell Biology

Department of Immunology, IMM-13

The Scripps Research Institute

10550 North Torrey Pines Road

La Jolla, California 92037, USA

Between Bench and Bedside provides the reader with a remarkable view of the culture of clinical experimentation in oncology, in particular, a view of the interactions between the biology laboratory and the oncology ward. In this book, a clinical trial of interleukin-2 (IL-2) begun in 1986 at the Cancer Foundation, a major French cancer treatment center, is critically examined in an attempt to answer the question "What can be learned from the study of a semiroutine clinical experiment in a cancer treatment center?" The author, an immunologist and science historian, observed the several small clinical trials comprising the Cancer Foundation's IL-2 trial from its be- specific pathogens, covering AIDS, malaria, schistosomiasis and Helicobacter pylori - an effective method to tie together general principles introduced in previous chapters. The topics are generally well selected, giving a representative overview of current challenges in vaccine design. A chapter addressing cancer vaccines, discussing both the similarities with immunization strategies aimed at infectious diseases and some of the unique challenges of antitumor immunization, would have strengthened this section. The closing chapter on vaccination therapy against autoimmune disease lucidly demonstrates the potentially broad applicability of modern vaccinology.

Overall, this book is a remarkable accomplishment. The reader is left with a clear impression that modern vaccinology is firmly founded in basic science advances from a number of disciplines, and that modern vaccine design is truly "rational" vaccine design. I would recommend this book to anyone with an interest in vaccine development. Those in this field who desire a broader perspective should find this book particularly useful.

ginning until July 1990. By using perspectives borrowed from the histories of science and medicine, the sociology of scientific knowledge, the ethnography of the laboratory and the sociology of medicine, she creates a very realistic picture of this and other clinical trials. She takes a detailed look at the collaborations between scientists and clinicians, two very different groups with a common goal to develop a cure for cancer.

Even though the narrative is occasionally patchy and heterogeneous, she offers a superb analysis in Chapters 1 through 3

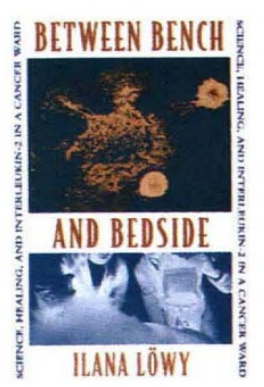

anomas. The overall response rate of IL-2 in the Cancer Foundation's trials was $20-25 \%$, which was similar to that reported in other trials in Israel and the United States. Even though these results were not as good as initially hoped, they nevertheless received favorable press coverage that lead, in 1993, to the creation of a clinical biology division that provided the nucleus for a new laboratory complex. It was hoped that this complex would eventually gather together analysis and clinical research laboratories, as well as laboratories dedicated to preclinical and industrial research.

In Chapter 5, the author describes in considerable detail the professional cultures, jurisdictions and practices that were involved in making the IL-2 trial work. She recounts how "material, social and discursive techniques, shared objects and concepts, control over resources and persons and the development of a specific 'intermediary zone' facilitated the development of efficient links" between the immunologists, oncologists and industrial IL-2 producers participating in this effort.

Finally in Chapter 6 the author summarizes the results of the trial and reflects on a "science-laden" pathology (that is, the direct dependence of therapeutic interventions in cancer on laboratory findings) between bench and bedside that leads to rich and complex interactions between a new medical practice, such as the introduction of IL-2, to cancer therapy and its "environment," that is, the other practices, claims and organizations that make up the culture of clinical experimentation in oncology. In the end, one can certainly agree of the historical and social background of the Cancer Foundation's IL-2 trial. The development of a distinct culture of clinical experimentation in the oncology clinics is clearly described, as is the application of immunological methods to cancer therapy and the specific story of IL-2 as an anticancer drug. The author's personal view of this clinical trial is both scholarly and realistic.

Administration of IL-2 alone or, in some trials, with either the patient's own lymphokine-activated killer cells or tumor-infiltrating lymphocytes to enhance the antitumor effect resulted in only a few "miracles" - complete and stable regression of disseminated mel- with the author's conclusion that as long as cancer is defined as a disease of "deviant cells" lacking an efficient treatment and as long as cancer therapy is structured as a strongly science-driven area of medical intervention, weakly dependent on life-style considerations, connections between the biology laboratory and the oncology ward will remain stable.

Between Bench and Bedside makes for excellent reading and can be highly recommended to immunologists and those among scientists and the public who seek to know more about the challenges involved in developing effective treatments for human malignancies. 